Trascender, Contabilidad y Gestión. Vol. 6, Núm. 16 (enero - abril del 2021).

Universidad de Sonora. Departamento de Contabilidad. México.

ISSN: 2448-6388. Reserva de Derechos 04-2015-04172070800-203.

\title{
El impacto de los programas sociales y el turismo en la reducción de la pobreza en el ámbito rural en México
}

The impact of social programs and tourism in reducing poverty in rural areas in Mexico

\author{
Luis Fernando Figueroa González ${ }^{1}$
}

Recibido: 19 de noviembre de 2020.

Aceptado: 14 de diciembre de 2020.

DOI: https://doi.org/10.36791/tcg.v0i16.95

JEL: M31. Mercadotecnia. L83. Turismo.

\section{Resumen}

México considerado un país del tercer mundo presenta una problemática bastante aguda en relación con la pobreza y la forma de vivir de las personas principalmente en el campo donde el crecimiento económico es limitado provocado por diversos factores. Para disminuir el nivel de pobreza sobre todo en el campo, el gobierno ha establecido una serie de programas encaminados a promover el desarrollo en el campo, para lo cual se ha considerado implementar el turismo en las comunidades tomando en cuenta los beneficios que esta aporta al implementarlas. De ahí la relevancia de saber que está haciendo el estado y las empresas privadas para hacer frente a este problema que se presenta principalmente en el campo mexicano. De ahí que el propósito de la presente investigación reflexiva vaya enfocado a conocer la efectividad de los programas del gobierno y de la actividad del turismo rural para reducir la pobreza en el ámbito rural de México. Para lo cual se revisó literatura de artículos científicos, páginas web, con temas relacionados con el tema que se ocupa en este documento. Algunos de los resultados encontrados fue que el Gobierno siempre ha instrumentado programas sociales y de desarrollo para el sector rural, dentro de los cuales se considera al turismo como alternativa de solución

\footnotetext{
${ }^{1}$ Luis Fernando Figueroa González. Licenciado en Administración. Doctor en Dirección y Mercadotecnia. Maestro de tiempo completo en la Licenciatura en Turismo, del Departamento de Contabilidad en la Universidad de Sonora. Correo: luis.figueroa@unison.mx
} 
a reducir la pobreza en el sector rural. Asimismo, se pudo concluir que el impacto de estos programas y la promoción del turismo rural no han dado los resultados esperados de su implementación.

Palabras clave: turismo, rural, programas, sociales, pobreza.

\section{Abstract}

Mexico, considered a third world country, presents a rather acute problem in relation to the poverty and the way of life of the people mainly in the field where economic growth is limited caused by various factors. To reduce the level of poverty especially in the countryside, the government has established a series actions at promoting development in the countryside, for which the development of tourism activity in the communities has been considered, taking into account the benefits that this contributes when implementing them. This is why it is important to know what actions are being developed by the government, as well as the private initiative, to be able to face the problem that rural communities suffer. Hence, the purpose of this reflective research is focused on knowing the effectiveness of government programs and rural tourism activity to reduce poverty in rural Mexico. For which the literature of scientific articles, web pages, with topics related to the subject that is dealt with in this document was reviewed. Some of the results found were that the Government has always implemented social and development programs for the rural sector, within which tourism represents an option solution to reduce poverty in the rural sector. Likewise, it was concluded that the benefits of these programs and the promotion of rural tourism have not yielded the expected results of their implementation.

Keywords: rural, tourism, social, programs, poverty.

\section{Introducción}

Un problema fuerte de la humanidad lo constituye la pobreza, fenómeno que se presenta en todos los países en diferentes grados o proporciones dependiendo del nivel de desarrollo de cada país. En este sentido México considerado un país del tercer mundo presenta una problemática bastante aguda en relación con la pobreza y el modo de vivir de sus habitantes, principalmente en las zonas rurales donde el crecimiento económico es limitado provocado por diversos factores. Para disminuir el nivel de pobreza sobre todo en el campo, el gobierno ha establecido una serie de programas encaminados a promover el desarrollo en el campo, para lo cual se ha considerado el turismo como acción en las comunidades tomando en cuenta los beneficios que esta aporta al implementarlas.

En las últimas décadas a nivel mundial las acciones para bajar el índice de pobreza han sido notorias, lográndose el objetivo de reducir a la mitad en 2010 el índice registrado en 1990, que estaba pronosticada para 2015, a pesar de esto la pobreza extrema a nivel mundial sigue siendo alta (Banco mundial, 2015) Si bien los índices de pobreza a nivel mundial han disminuido, esto no se ha dado en forma uniforme en virtud de que algunos áreas o países han logrado reducir drásticamente sus tasas de pobrezas como Asia Oriental y China, todavía existen muchos países donde su principal problema es la pobreza extrema, principalmente en los países subdesarrollados donde se presentan situaciones 
como es un lento desarrollo económico, alto desempleo, migración elevada a las ciudades (Figueroa, 2012). Aunado a esto la situación precaria de algunas comunidades rurales en el mundo es compleja.

Según estadísticas en muchos países subdesarrollados las personas que viven en el campo tres de cada cuatro son pobres (Banco Mundial, 2007) donde la mayoría subsisten con ingresos muy bajos los cuales provienen de la actividad de la agricultura (Colino, Gómez y Martínez, 2014).

En 2012 el número que de personas que vivían en el campo representaban el $23.1 \%$ de la población total de México, alrededor de 26 millones de personas donde los índices de pobrezas son más grandes que los que se presentan en las zonas urbanas como se muestra en la tabla 1, donde las mujeres es el sector más afectado.

\section{Tabla 1}

Distribución de la pobreza en México

\begin{tabular}{|l|r|r|r|}
\hline Zona & \% pobreza & \multicolumn{1}{|c|}{$\begin{array}{c}\text { \% pobreza } \\
\text { extrema }\end{array}$} & \multicolumn{2}{c|}{$\begin{array}{c}\text { \% pobreza } \\
\text { moderada }\end{array}$} \\
\hline Rural & 61.6 & 21.5 & 40.1 \\
\hline Urbana & 40.6 & 6.3 & 34.3 \\
\hline
\end{tabular}

Fuente: Diario Oficial de la Federación-DOF 2013.

\section{El papel del estado en la pobreza}

Las acciones que el estado realiza para atender a los más vulnerables debe ser acordes al contexto de cada país, considerando la información con que se cuenta y las prioridades de las personas. Para poder reducir la pobreza, los países necesitan considerar algunas medidas entre las que se pueden mencionar: a) generar fuentes de empleo; b) desarrollo humano principalmente en aquellos que puedan acceder a servicios básicos, y c) ayudar a las personas en situación vulnerable ante situaciones que puedan influir aún más en la pobreza, pandemias, inestabilidad de precios en los alimentos (Banco Mundial, 2015).

México no es la excepción ante el problema de la pobreza la cual para tratar de reducirla el Estado ha desarrollado una serie de políticas sociales encaminadas a lograr una asignación más equitativa de los recursos en los grupos más vulnerables y marginados. En este sentido según Cortez, Juárez y Ramírez (2011) la política social tiene como objetivo reducir la pobreza para lo cual una de las acciones ha sido la formación de la Secretaria de Desarrollo Social (Sedesol), la cual coordina diversas subsecretarias que ejecutan programas sociales. Algunos de estos programas de asistencia social son el Programa Nacional de Solidaridad (Pronasol) creado en 1988, el cual coordino los programas PIDER (Programa de inversiones públicas para el desarrollo rural), y COPLAMAR (Coordinación General del Plan Nacional de Zonas Deprimidas y Grupos Marginados). En la década de los 90’s surge el programa PROGRESA que al igual que los programas precedentes tenía como objetivo reducir la pobreza mediante el subsidio de servicios básicos para la 
población. Dicho programa para la década del 2000 cambia de nombre a de OPORTUNIDADES, siendo el instrumento más importante del estado mexicano para combatir la pobreza, y en 2020 Pensión para el Bienestar de las Personas Adultas Mayores, entre otros.

\section{El turismo como opción para combatir la pobreza}

En un mundo globalizado y competitivo, es importante conocer los factores que influyen en la conducta del consumidor para adquirir determinado producto o servicio, dentro de los cuales se encuentra el turismo donde un aspecto importante son las motivaciones que las personas tienen para tomar una decisión. En el campo del turismo resulta de vital importancia conocer los factores que influyen en las personas al tomar su decisión de viajar, la elección de un lugar específico y el grado de satisfacción obtenido (Fernández, 2008).

El turismo se considera una actividad económica ya que produce los elementos para satisfacer necesidades. Las actividades económicas se clasifican en tres sectores que son: primario relacionado con la naturaleza, secundario que se relaciona con la industria de transformación, y el terciario que considera la actividad de los servicios. En base a la clasificación anterior, la actividad del turismo se ubica dentro del sector terciario, el cual se puede definir en forma concreta como: "el desplazamiento de la gente por diversas causas al menos por un día y menos de un año, tanto dentro como fuera del país” (Guzmán y Rebolloso, 2012 p. 72).

El turismo es una actividad que tiene mucha influencia en la economía a nivel mundial, ya que representa una fuente generadora de ingresos, empleo y desarrollo para muchos países, contribuyendo además con el desarrollo de las personas, así como en el aspecto económico y social a través del entretenimiento generando una interacción de los turistas con las personas de los destinos turísticos. (Guzmán y Rebolloso, 2012).

Como actividad económica el turismo ofrece una serie de alternativas a los visitantes como es la estancia, cultura, historia, patrimonio y la naturaleza que coadyuvan a la recreación y entretenimiento. De igual forma contribuye a la generación constantes de ingresos por su elevado desarrollo (Rogel, 2011).

El desarrollo del turismo trae como resultado diversos beneficios económicos y sociales para los lugares de destino. El crecimiento de esta industria por lo regular viene acompañada de algunos costos afectando la vida de los habitantes de las comunidades. Al mismo tiempo el éxito del turismo depende del soporte activo de las gentes de los lugares a visitar, quienes deben poner atención en el impacto que tendrá al turismo en el aspecto sustentable y considerarse este al momento de hacer una planeación de actividades turísticas. Otros de los beneficios que el turismo proporciona son las oportunidades de empleo, fortalece la economía local, contribuye a incrementar el estándar de vida, y proporciona oportunidades de inversión. De igual forma incrementa las instalaciones recreativas, enriquece los valores culturales y eleva la calidad de vida de las personas (Nunkoo y Ramkissoon, 2011).

Debido a la variedad de atractivos turísticos que México ofrece como: sol y playa, cultural, y alternativo o de naturaleza; esto ha permitido que una gran afluencia de personas extranjeras lo visiten, provocando con ello que se le situé dentro de los países líderes del turismo, ya que está considerado dentro de los 10 países más importante del mundo en relación con el turismo, 
esto gracias a las políticas manejadas a través del Plan Nacional de Desarrollo Turístico implementado en la década de los sesenta, generando con ello el aprovechamiento de destinos turísticos como Cancún, Los cabos entre otros. Esto ha permitido que el turismo sea un factor importante en la generación de empleos representando el 9\% del Producto Interno bruto. (Benseny, 2007).

Con base en el Plan de Desarrollo Turístico, se han podido realizar una serie de acciones encaminadas al desarrollo de múltiples áreas rurales mediante la generación de actividades adicionales a las que realizan normalmente en el campo (Garduño, 2009). Desafortunadamente se destinan bajos recursos económicos para desarrollar el turismo rural por lo que el aprovechamiento de 127 áreas protegidas con cuenta México cuenta no han podido ser desarrolladas. (Juárez y Ramírez, 2007).

Lo anterior debido a que diversos programas que maneja el estado Mexicano a través de SECTUR se enfocan más en reforzar zonas rurales posicionadas como son: tesoros coloniales y pueblos mágicos por mencionar algunos, dejando por un lado muchas áreas rurales con atractivos turísticos que no prosperan por falta de información y de apoyos, considerando que el turismo es un elemento clave para el crecimiento económico que bien contribuir a minimizar la pobreza en el sector rural con la comercialización de productos turísticos como son los recursos naturales y culturales. (López- Guzmán, 2009).

Aunado a lo anterior la situación precaria de algunas comunidades rurales en el mundo es compleja. Coinciden diversos factores tales como destinar recursos económicos para hacer funcionar su economía, lo que ha hecho que los trabajos tradicionales del campo sean diversificados por otros para contribuir a elevar el poder adquisitivo de los habitantes de las comunidades rurales y puedan con ello tener una vida de mejor calidad (Ruiz y Barroso, 2011).

Por lo que resulta relevante saber que está haciendo el estado y las empresas privadas para contrarrestar los indicadores de pobreza que se presentan en el contexto rural. Aquí es donde el turismo mediante acciones concretas puede significar una opción para contribuir al crecimiento rural mediante la creación de una buena oferta turística (Talaya, 2010), logrando con su comercialización el flujo de ingresos adicionales al trabajo tradicional de campo que normalmente realizan.

El gobierno Mexicano como responsable principal de reducir la pobreza en todos los ámbitos pero principalmente en el campo, ha diseñado diversos programas con el fin de desarrollar las economías rurales con actividades distintas a las tradicionales que se llevan a cabo en el campo como es el turismo, lo cual se implementó mediante el programa del Fondo Nacional de Apoyo a Empresas Sociales (FONAES) que durante los años 90's, apoyo a estados con una población indígena alta y en situación de pobreza. El turismo permitió crear un alto número de empleos permanente y temporales mediante la ejecución de 30 proyectos en áreas rurales (Garduño, Guzmán y Zizumbo, 2009).

Considerando lo anteriormente expuesto, el propósito de esta investigación es conocer la efectividad de los programas del gobierno y de la actividad del turismo rural para reducir la pobreza en el ámbito rural de México.

\section{Revisión de literatura}

\section{El turismo rural}

El turismo rural representa una opción distinta para las personas que gustan de hacer un turismo diferente al 
tradicional y que buscan de experimentar nuevas emociones interactuando con personas $\mathrm{y}$ lugares desconocidos (Pérez, 2010), y si se toma en cuenta que actualmente muchas personas buscan algo nuevo que los saque de su rutinas habituales de la ciudad buscaran destinos tranquilos con culturas diferentes y con atractivos naturales (García, 2005); lo que significaría para este tipo de destinos turísticos, una oportunidad para crear negocios mediante la oferta de productos y servicios desarrollando con esto su economía local mediante la diversificación de ingresos adicionales a los generados por el trabajo de campo que realizan (Garduño, 2009).

De igual forma es un sistema productivo alterno por la oferta de diversos atractivos turísticos mediante los cuales los habitantes de una comunidad obtienen más ingresos, $\mathrm{y}$ en ese sentido representa una alternativa adicional al trabajo de campo ya que contribuye al crecimiento y aprovechamiento de productos turísticos opcionales lo que permite definirlo como: "diversidad de actividades ofrecidos por los habitantes de una comunidad con el fin de provocar el flujo de personas logrando con ello ingresos adicionales (Pérez, 2010, p. 5). En forma más completa puede decirse que el turismo rural considera también la historia, costumbres, folclor, tradiciones y patrimonio (MacDonald y Jooliffe, 2003).

En los países en desarrollo el turismo rural juega un papel muy importante en virtud de que contribuye a elevar sus economías y reducir la pobreza en esos lugares apartados de las ciudades, es por ello que algunos organismos como el Banco Mundial y el Fondo Interamericano de Desarrollo Agrícola entre otros han estado llevando a cabo programas que permitan el desarrollo del turismo rural el cual otra forma de definirlo es: “opción de viaje para las personas hacia el campo con el fin de estar en contacto con el campo y realizar actividades y deportes en interacción con la naturaleza como es el ecoturismo, turismo de naturaleza, turismo de aventura que contemplan diversas actividades entre las que se pueden mencionar la caza, la pesca, conocimientos culturales e históricos entre otras" (Millán, 2006; Solsona, 2006).

Es por ello que el turismo rural permite que ingresos adicionales fluyan hacia las comunidades mediante las actividades antes mencionadas y con la prestación de servicios como son el hospedaje, gastronomía, estaciones de gasolina por mencionar algunos. Toda esta diversificación de productos y servicios permiten que el turismo rural contribuya al desarrollo de las economías rurales las cuales inciden en una mejor forma de vivir de los habitantes de las comunidades (Millán, 2006; Diéguez, 2009).

Si se toma en cuenta que las actividades agrícolas no contribuyen a satisfacer todas las necesidades y que por lo mismo no permite que las personas tengan una mejor forma de vivir, el turismo rural se convierte en una herramienta para realizar toda una serie de acciones para apoyar poblaciones que se encuentran desatendidas por el sector público y privado y a la vez contribuyen a que se disminuya la dependencia del trabajo del campo (Garduño, 2009; Pérez, 2010).

Además del aspecto económico el turismo rural se convierte en un factor importante para fomentar los aspectos culturales y sociales, así como la conservación del entorno ambiental de las comunidades, por lo que resulta importante crear un lazo entre los diferentes factores que contribuyen al desarrollo rural para estar en posición de ofrecer alternativas de recreación a las personas que gustan de hacer turismo de una forma diferente (Serrano, 2010). 
Una forma de lograr lo antes mencionado es aprovechar los recursos naturales, culturales y patrimoniales con que cuentan las comunidades rurales y diseñar productos y servicios que se puedan ofrecer en tiempos razonables con el fin de elevar el desarrollo sobre todo económico, pero sin descuidar el cuidado del medio ambiente, lo cual se convertiría en algo distintivo de cada comunidad dependiendo de los recursos con que cuente (Mazaro y Varzin, 2008). Lo que trae como consecuencia una mayor sensibilidad hacia el aspecto sustentable que llamaría el interés de las personas por visitar destinos ambientales que no degraden el entorno natural y cultural, como pueden ser los parques temáticos y los mega centros turísticos que combinan actividades lúdicas con el esparcimiento (Rodríguez y Pallerols, 2003). En este sentido los atractivos naturales están siendo demandados cada vez más por los turistas, por lo que mantener su sostenibilidad, los convierte en un producto turístico que satisface la necesidad de ocio y esparcimiento (Strickland-Munro, 2010).

\section{Turismo sustentable}

Algunos conceptos sociales y naturales están influyendo en la nueva forma de hacer turismo, y que se están analizando desde diferentes puntos de vista para establecer lo que es la Sustentabilidad, concepto que se integrado al turismo y que básicamente es un principio de orden para el desarrollo sustentable, que permite medir el impacto económico, social y sobre todo ambiental del turismo en el medio rural (Osorio, 2005; Walligo, 2012).

El desarrollo sustentable fue definido por un informe llamado ONU-Brundtlans como (Rigall-I-Torrent, 2008 p. 884): "forma de cubrir las necesidades de las personas tanto en el presente como en el futuro" Este concepto sirvió de base para que la Organización Mundial de Turismo [OMT] definiera el turismo sustentable de la siguiente manera (Pinilla, 2009, p. 152): “actividad encaminada que permite cubrir las necesidades de los turistas y de los lugares de destino protegiendo y fomentando en forma paralela las opciones a futuro". Considerando lo anterior se puede apreciar la estrecha relación que existe entre el turismo el cuidado del medio ambiente considerando que el turismo sustentable es un tipo de turismo que intenta evitar daños al medio ambiente (Ortiz y Camargo, 2010).

Actualmente algunos investigadores han establecido que no existe una definición exacta de turismos sustentable, en virtud de que para ello se considera el punto de vista y la ideología, por lo que el turismo sustentable es considerado de diversas formas entre las cuales se puede mencionar: es un turismo el cual es económicamente viable, sin destruir los recursos los cuales representan el turismo en el futuro principalmente en el medio ambiente y las interacciones sociales de la comunidad y sus habitantes (Saarinen, 2006). De igual manera el turismo en sus formas de convencional y alternativo, es compatible con el desarrollo sostenible cual no necesariamente implica crecimiento, sino más bien una realización social y económica, que mejora la forma de vivir de una comunidad y sus habitantes (Liu, 2003).

\section{Características del Turismo Sustentable}

Algo que distingue la sustentabilidad en el turismo es que deja de ser estacional para ser permanente, para lo cual se debe hacer un análisis ambiental teniendo como objetivo satisfacer necesidades en todo momento, esto mediante una serie de actividades enmarcadas en el concepto de sustentabilidad tomando en cuenta la relevancia del cuidado ambiental. Esto puede ser posible mediante el diseño de un modelo que permita generar algunos beneficios económicos sin descuidar aspectos como el cuidado ambiental y la conservación de 
tradiciones, y contribuyendo al desarrollo social. (Ortiz y Camargo, 2010). Para lograr esto es necesario considerar que los recursos naturales tienen caducidad y que lleva tiempo que se regeneren, razón por la cual el acceso a ellos es escaso debido al mercado exigente que son los turistas (Portal, 2008).

Otra distinción del turismo sustentable, es el papel que el gobierno desempeña para fomentar su desarrollo a través de una serie de medidas regulatorias de los recursos naturales, así como la supervisión constante de los ingresos que genera el turismo en un ambiente de libre mercado (Torres-Delgado y López, 2012). Estas medidas comprometen a los actores del turismo a ofertar productos turísticos que cumplan con las expectativas de los turistas por el precio que están dispuestos a pagar, además establecen medidas enfocadas al cuidado ambiental lo que representa una ventaja diferencial en un mercado tan competido como es el turismo (Zhang, 2009).

De igual forma, el turismo sustentable considera tres aspectos importantes que componen la sustentabilidad que lo caracteriza, siendo estos componentes: sustentabilidad económica, ambiental y socio-cultural, y en los cuales tiene un impacto positivo ya que eleva la economía, cuida el medio ambiente e involucra a los principales actores en su desarrollo (Cardoso, 2006).

\section{Importancia del Turismo Sustentable}

La industria del turismo hace una fuerte contribución al desarrollo sustentable, en el aspecto de la generación de empleos, sobre todo para las mujeres que habitan en áreas rurales marginadas, procurando no perjudicar el medio ambiente (Fortanier y Van, 2010). Asimismo, el turismo sustentable para su desarrollo considera el uso de los recursos escasos como la tierra, mano de obra, energía, que se requieren para la conservación de la naturaleza, fomentando con esto un nuevo estilo de vida, al diversificarse las actividades de las comunidades en este proceso contribuyendo con ello a una mejor calidad de vida (Tao y Wall, 2009).

Otro punto importante del turismo sustentable (TS) implica su orientación hacia el encuentro de las necesidades de una comunidad, garantizando la protección del medio ambiente, satisfaciendo la demanda turística, manteniendo la atracción del lugar independientemente del aumento o disminución de dicha demanda, sin descuidar el aspecto económico que beneficia a todos los actores en esta actividad (Tarlombani, 2005). Y son los mismos residentes quienes deciden si se vuelven dependientes del turismo sustentable asumiendo los costos y beneficios que esto implica por la preocupación de la conservación de entorno (Lee, 2013).

El TS permite establecer restricciones físicas en cuanto al desarrollo de las actividades turísticas, con el fin de conservar una mejor forma de vida de las comunidades a largo plazo (Guzmán y Rebolloso, 2012). Esto es posible gracias a que es factible de planearse de tal forma que los turistas ayuden a promover y apoyar la conservación y regeneración de los recursos del entorno (Connell, 2009). Además, la implementación de sistemas de administración sustentable permite conservar áreas críticas naturales, equilibrar la demanda turística para el desarrollo apropiado y estimular a las comunidades mediante su crecimiento económico y la preservación de sus áreas naturales (Ghaderi y Henderson, 2012).

\section{El Turismo Sustentable y la pobreza}

Un aspecto importante del TS es que contribuye a 
minimizar la pobreza principalmente en comunidades rurales, en virtud de que enfoca su interés en la satisfacción de las necesidades de una comunidad mediante una mejor forma de vida en el mayor tiempo posible, de igual forma satisface las demandas de los turistas preservando el ambiente natural, buscando con esto un balance entre lo económico y lo ambiental (Hunter, 2002; Liu, 2003). Pudiendo generar con esto una serie de beneficios para una comunidad como es la distribución justa de costos y beneficios, generar empleos, influye en la creación de infraestructura en las comunicaciones, y fortalece la identidad cultural (Tarlombani, 2005).

De igual forma permite la creación de recursos económicos para la protección ambiental, mediante una normatividad para el comportamiento del cuidado de la naturaleza tanto por parte de los turistas como de los habitantes de una comunidad (Vázquez, 2004). En este sentido los prestadores de servicios contribuyen en la sensibilización de las habitantes mediante el ejemplo al desarrollar éticamente al manejar productos y servicios con costos y calidad acorde con la calidad y una realidad económica (Lee, 2013), logrando con esto la optimización de los recursos naturales que permiten el desarrollo del turismo derivando en beneficios socioeconómicos a todos los que intervienen en su desarrollo (Rigall-I-Torrent, 2008).

\section{El turismo rural y la sustentabilidad}

La creciente demanda de las personas por hacer un turismo distinto al tradicional, está generando que se oferten más productos y servicios sobre todo porque, las estadías son más cortas. (Nolte, 2008). El concepto de la sustentabilidad en el turismo es que considera el impacto ambiental y la forma en que se maneja para poder tener un crecimiento sostenible (Saarinen, 2006). Es por ello que resulta de gran importancia la participación de los habitantes de las comunidades mediante el cuidado adecuado de los recursos naturales con que cuentan, para lograr su conservación (Bruyere, 2009).

De igual forma los efectos sobre el medio ambiente que tiene el turismo sustentable durante su desarrollo, se ha logrado reducir por la nueva cultura de la gente en el sentido de lo que representa el cuidado de la naturaleza (Touraya, y Junga, 2010). Lo anterior obliga a que el Turismo Rural Sustentable (TRS) tenga como objetivo reducir el impacto ambiental para lograr un desarrollo económico mediante la correcta interacción de los visitantes, y los proveedores de bienes y servicios con las comunidades a visitar (Lane, 2005). Es por ello que todo tipo de turismo debe ser sustentable en todas sus formas para que pueda contribuir al desarrollo sustentable (Pinilla, 2009).

Lo anterior es consecuencia de la alta demanda que tiene el turismo distinto al tradicional y que está generando sensibilidad en los turistas en cuanto a su comportamiento en el medio natural (Nolte, 2008).

\section{Participantes en el turismo rural}

El turismo rural sustentable, permite la organización de comités locales para promover el desarrollo de esta actividad mediante la conservación del entorno natural. Estos comités por lo regular son formados por miembros de las diversas organizaciones que intervienen como actores en la actividad del turismo, logrando con ello una integración social, ambiental y económica (Yasarat, 2010). Es por eso que resulta importante la participación de los habitantes de las comunidades en esta nueva forma de trabajo con el fin de que se pueda diseñar una oferta turística con los recursos con que se cuenta, sin descuidar los efectos sociales, económicos y ambientales (González, 2011). 


\section{Los habitantes de las comunidades}

La participación de los habitantes de una comunidad en el desarrollo de proyectos turísticos es de gran relevancia para que se pueda elevar la calidad de vida de una comunidad, al tener acceso a los beneficios que se derivan del TR en lo económico principalmente, social, cultural y natural. Es por ello que se debe considerar la inclusión de las personas del lugar del destino, ya que además de ser un derecho que se tiene al participar en la planeación, representa una oportunidad de desarrollo personal y familiar (Prabhakaran y Ramachandran, 2014).

Sin embargo el nivel de participación de las personas de una comunidad rural en el desarrollo del turismo es en un nivel muy bajo, en virtud de que generalmente esta actividad es realizada por persona u organizaciones especificas quienes están organizados de tal manera que manejan y coordinan las actividades así como los beneficios que el TR aporta a una comunidad, razón por la cual a muchos de sus habitantes no les interesa participar por sentirse excluidos por parte de estos grupos además de que no cuentas con los recursos esenciales para detonar proyectos turísticos en sus comunidades. (Forstner, 2004).

En México el gobierno promueve el turismo comunitario como una forma de involucrar a la población local de las comunidades en la gestión turística. Esta nueva forma de trabajo es considerada como una estrategia para desarrollar el bienestar de una población a través de una justa repartición de los beneficios económicos, la valoración de los recursos naturales y la identidad cultural (Rodríguez y Acevedo, 2015).

La educación y/o preparación de los habitantes de una comunidad para realizar actividades relacionadas con el turismo juegan un papel muy importante, y de acuerdo con Díaz y Fernández (1995), es reducido el número de habitantes de una comunidad que pueden ser involucrados en los proyectos turísticos, si se toma en cuenta que la mayoría no cuenta con una preparación para involucrarse en este tipo de actividades, quedando únicamente la opción de trabajar en las empresas proveedoras de los servicios turísticos las cuales solo pueden contratar un número muy reducido de personas, por lo que el resto de una comunidad solo pueden participar fabricando y comercializando en forma personalizada productos típicos, haciéndolo de una manera informal de acuerdo a lo que su instinto le marca como es la amabilidad, el convencimiento entre otras formas. La efectividad de la comercialización de sus productos podría ser más positiva si se pudiera complementar su trabajo con una educación más formal que sumado a la experiencia le da en el trabajo diario de la venta, podría elevar su participación en el turismo rural.

En la tabla 2 se pueden apreciar habilidades informales que se desarrollan en el trabajo diario de venta, y los conocimientos formales que se pueden adquirir para mejorar el desempeño como microempresarios en el turismo rural. 
Tabla 2

Habilidades formales e informales para la comercializar de productos turísticos en forma individualizada

\begin{tabular}{|l|l|}
\hline \multicolumn{1}{|c|}{ Habilidades informales } & \multicolumn{1}{c|}{ Habilidades formales } \\
\hline - amabilidad & $\bullet$ trabajo en equipo \\
$\bullet \quad$ capacidad de persuasión & $\bullet$ responsabilidad \\
- carisma & $\bullet$ comunicación \\
- creatividad & $\bullet \quad$ actitud de servicio \\
\hline
\end{tabular}

Fuente: Elaboración propia.

\section{Intermediarios}

Muchas comunidades enfrentan problemas similares como es la comercialización de sus productos, ya que dependen en gran parte de diversos intermediarios como son las empresas privadas, organismos del sector público y organizaciones no gubernamentales, quienes son los encargados de realizar dicha comercialización, ya que cuentan con expertos y experiencia en diversos campos como es el conocimiento del marketing, recursos financieros, capacidad de construir y otros conceptos que impactan la forma de vivir de los habitantes de una comunidad. Si bien el turismo es una actividad que puede ayudar a reducir la pobreza en el medio rural, este tiene efectos sobre todo en la transformación sociocultural y física de una comunidad, y muchas veces la ejecución por parte de agentes externos como son los intermediarios, puede provocar implicaciones mayores que los beneficios esperados por una comunidad (Forstner, 2004: Madrigal-Uribe, 2011).

Los intermediarios del TR regularmente no tienen la intención de apropiarse de los recursos con que cuenta un comunidad para desarrollar productos turísticos, sino que los aprovechan para lograr una mejor forma de vida de los habitantes en lo económico, sociocultural y natural, sin embargo frecuentemente solo favorecen a un reducido grupo de una comunidad con los beneficios que el TR trae consigo, lo cual lejos de resolver los problemas de pobreza del campo, en muchos casos no se considera a las personas de una población, son excluidos de los proyectos turísticos. Es por ello que el TR en muchos casos puede ser manipulado en términos sociales, culturales y en deterioro del medio ambiente impidiendo de esa forma la participación de los habitantes de una comunidad en los planes de un proyecto turístico, y por lo tanto sin acceder a los beneficios económicos que dé él se derivan. (Pérez, 2011; Prabhakaran y Ramachandran, 2014).

Considerando el papel que juegan los intermediarios en la promoción del TR, se pueden mencionar algunas ventajas y desventajas que los caracterizan como por ejemplo las que se muestran en la tabla 3. 


\section{Tabla 3}

Ventajas y desventajas de los intermediarios en el TR

\begin{tabular}{|l|l|}
\hline \multicolumn{1}{|c|}{ Ventajas } & \multicolumn{1}{c|}{ Desventajas } \\
\hline $\begin{array}{l}\text { Manejan información de mercado y tienen } \\
\text { experiencia. }\end{array}$ & $\begin{array}{l}\text { Están interesados principalmente en su beneficio } \\
\text { propio. }\end{array}$ \\
\hline $\begin{array}{l}\text { Promueven la calidad de productos y lugares de } \\
\text { destino. }\end{array}$ & $\begin{array}{l}\text { No toman en cuenta a los habitantes en el } \\
\text { desarrollo de proyectos turísticos. }\end{array}$ \\
\hline $\begin{array}{l}\text { Tienen capacidad de invertir en infraestructura } \\
\text { local }\end{array}$ & $\begin{array}{l}\text { Las utilidades económicas no la reinvierten en la } \\
\text { comunidad. }\end{array}$ \\
\hline Establece canales de distribución & No existe una coordinación con la comunidad. \\
\hline $\begin{array}{l}\text { Pueden apoyar financieramente para el desarrollo } \\
\text { de proyectos turísticos. }\end{array}$ & $\begin{array}{l}\text { Desconfianza con los proveedores locales de } \\
\text { servicios. }\end{array}$ \\
\hline
\end{tabular}

Fuente: Elaboración propia con información de Forstner, 2004.

De acuerdo con Adeyinka-Ojo, Khoo-Lattimore y Nair, (2014), una forma de evitar la manipulación y los manejos del turismo rural en beneficio de un reducido número de participantes de los cuales en diversas situaciones las persona de una población son excluidos, es a través del establecimiento de una oficina que trabaje en coordinación con los principales actores en el desarrollo de proyectos turísticos administrando y promoviendo el turismo de una comunidad mediante diversas estrategias de marketing, desarrollando actividades como son: proporcionar información a los visitantes, diseñar una imagen rural, realizar convenios, organizar campañas publicitarias. Sin embargo, este tipo de oficinas han sido subestimadas para su operación en áreas rurales, a pesar del importante papel que pueden jugar en la promoción de un lugar de destino rural.
En este sentido en México se creó la Oficina de Visitantes y Convenciones (OCV'S) las cuales pueden ser públicas, privadas o no gubernamentales y cuya responsabilidad es la promoción y posicionamiento de un destino. Con el fin de desarrollar actividades principalmente de marketing para lograr su propósito, reciben financiamiento mediante el impuesto del $2 \%$ al hospedaje el cual es distribuido a través de los gobiernos estatal y municipal para distribuirlo en las distintas OCV's que funcionan en el estado (Ramírez y De las Hera, 2013). Sin embargo, en entrevistas personales realizadas a operadores de este tipo de oficinas en el Estado de Sonora, México, mencionaban que dichos recursos no se les proporcionaba por parte del sector gobierno, lo que hace imposible la promoción del turismo en las ciudades donde operan incluyendo una comunidad rural. 


\section{El sector publico}

En forma generalizada se puede decir que los gobiernos ven en el TR una alternativa para reducir los índices de pobreza en el campo, para lo cual la mayoría apoya a las comunidades para el desarrollo de actividades turísticas o bien en la promoción de los lugares de destino resaltando los atractivos naturales con que cuentas dichas comunidades. Sin embargo es muy raro que inviertan en el desarrollo de proyectos turísticos rurales (Forstner, 2004). En México es indudable que los gobiernos en sus tres niveles así como sus instituciones han contribuido al impulso de esta actividad como es la SECTUR con su programa de Pueblos Mágicos, pero bajo directrices que marcan organismos internacionales los cuales son en parte fuente de financiamiento para el desarrollo de esta actividad, y en la mayoría de los casos estos financiamientos no son aplicados en forma adecuada lo que provoca una desviación destinando tan solo una parte para el impulso de la comunidades rurales a través del turismo (Madrigal-Uribe, 2011).

Sin embargo, a pesar de una administración inequitativa de los recursos para fomentar el turismo rural, México a través de sus programas y de las normas oficiales en materia turística ha logrado mantener una ventaja competitiva que le está permitiendo ser una potencia en turismo a nivel mundial situándose en 2014 en el lugar 15 (Ibañez, 2015).

\section{El turismo rural integrado}

Una nueva forma del turismo rural muy diferente al tradicional es el Turismo Rural Integrado (TRI), el cual considera a los participantes en la promoción del turismo donde además de establecer una coordinación entre ellos, establece responsabilidades ligadas con la realización de acciones en el seguimiento y administración con lo que se cuenta (Saxena et al., 2009). Esto permite establecer diversas formas de relación entre los participantes como son los turistas, empresarios locales, y empresas que promueven el turismo y por supuesto las personas de la población (figura 1), de no lograrse estas relaciones difícilmente se lograrían concretar la realización de acciones o estrategias (Cawley y Gillmor, 2007).

\section{Figura 1}

Actores del Turismo Rural Integrado

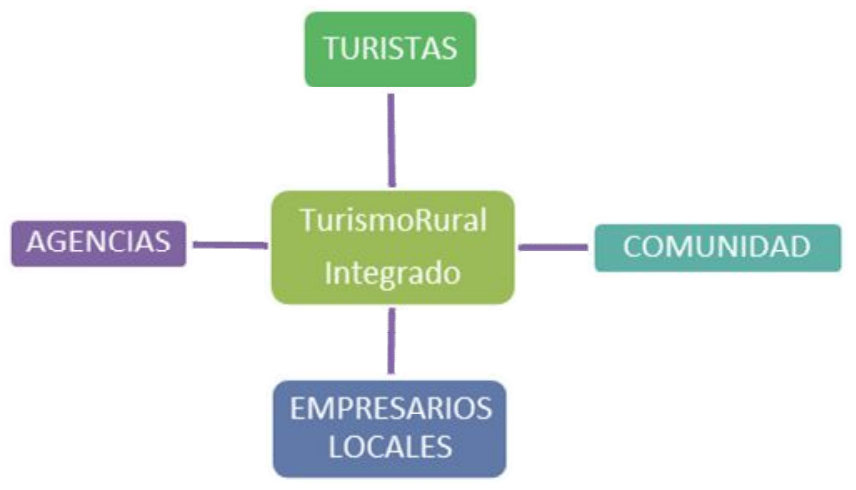

Fuente: Cawley y Gillmor, 2007. 
Esta relación entre los que participan en el Turismo Rural constituye una total integración entre ellos y las comunidades para hacer posible una realización real del turismo, logrando con ello con lo que se conoce como Turismo Rural Integrado el cual permite que todos los participantes conozcan el papel que desempeñan, así como las responsabilidades que ello conlleva (Petersen, 2010). Para lograr esta nueva forma de trabajo es necesario diseñar estrategias entre los participantes para proponer soluciones como son: identificar oportunidades, reducir las diferencias entre ello; planear el manejo de los recursos en forma sustentable, $\mathrm{y}$, lograr acuerdos entre instituciones (Saxena, 2007).

La integración como un concepto se está utilizando mayormente en la planeación principalmente referente a la sustentabilidad, pero requiere de la interacción entre varios niveles de entre las organizaciones y los diversos actores para realizar asociaciones horizontales y verticales, reduciendo las fricciones que se pudieran presentar y alcanzar de esta forma una integración que ayude a lograr estrategias consensuadas (Panyik, 2011).

Dentro del Turismo Rural Sustentable se ha dado énfasis a la integración del medio ambiente a esta industria, así como a la economía y la cultura, y recientemente la participación de la comunidad la cual se refleja en el porcentaje de gente empleada, tipos y grados de decisión, así como la intervención de los dueños de los recursos turísticos locales, estableciéndose un nexo de relaciones con el turismo rural (Saxena, 2008). De esta forma la actividad del turismo se integra a las economías locales y regionales generando beneficios directos de ingresos a las comunidades apoyándose en la asociación, la sinergia y la participación (Saxena, 2007).

Una forma de lograr mayor efecto en el TRI es dar mayor importancia a lo que es la sustentabilidad y los elementos que la constituyen, así como a las personas que en ella participan, mediante una implementación de políticas que vayan encaminadas a la par con el turismo, y así poder obtener un crecimiento sustentable (Cawley y Gillmor, 2007). Al considerar todos estos aspectos en el desarrollo de una buena planeación se podrán obtener los beneficios que del turismo se derivan asegurando con ello el correcto funcionamiento operacional del TRI (Petersen, 2010).

\section{Limitantes del turismo rural para reducir la pobreza}

Si bien se ha mencionado la gran variedad de beneficios que el turismo ofrece a las comunidades rurales para que puedan elevar su calidad de vida, también se ha comentado que eso sucede solo en ciertas comunidades que ya están identificadas como destinos turísticos rurales y que por lo tanto son consideradas dentro de los programas de apoyo que el gobierno tiene para impulsar el desarrollo de esta actividad (LópezGuzmán, 2009). En este sentido todavía quedan cientos o miles de comunidades que no han sido aprovechados los recursos con que cuentas para desarrollar una tan atractiva como es el turismo. De acuerdo con Solsona (2014) esto debido a una serie de causas que impiden implementar actividades turísticas como son:

- El área geográfica. - donde los recursos naturales y culturales, sufren de un deterioro en el paisaje por no haber sido utilizados o aprovechados, la contaminación ambiental provocada en la mayoría de los casos por las personas, pero también por desastres naturales, la existencia de infraestructura deficiente como son los caminos de acceso y los medios de transporte apropiados, así como la existencia de suelos que no son compatibles con actividades turísticas. 
- La población. - En este sentido se presentan una serie de limitantes como son la falta de motivación e iniciativa, el envejecimiento de la población, la falta de preparación para el desarrollo de la actividad del turismo.

- Los productos. - se considera como limitante no solo la falta de desarrollo y oferta de productos de productos locales en forma, sino también la carencia de servicios públicos, lo cual genera un bajo nivel competitivo con otras comunidades.

- Gestión turística. - en la mayoría de las comunidades solo se da por estacionalidad como son las fiestas religiosas entre otras, asimismo la falta de organización de los órganos gestores del turismo y la escaza comunicación entre los mismo.

Todo esto impide que muchas comunidades rurales puedan desarrollar actividades turísticas para elevar su calidad de vida, y por lo tanto la pobreza y la migración seguirán siendo las características de muchas poblaciones rurales en México mientras los diversos actores públicos y privados no se interesen por el desarrollo del turismo en el ámbito rural planeando e implementando acciones encaminadas a subsanar las limitantes mencionadas anteriormente.

\section{Críticas de los programas y del turismo rural}

El turismo rural a pesar de los beneficios que deja a una comunidad, es criticado por el impacto que este tiene sobre todo en el medioambiente y los conflictos que genera entre los promotores de esta actividad incluyendo a los habitantes de un lugar de destino.

Siguiendo a Shu-Tsu (2010), muchos países han optado por el desarrollo del turismo como una alternativa para incrementar los ingresos de las personas mediante la generación de empleos y con ello elevar la calidad de vida de los residentes. Sin embargo, esta actividad ha sido objeto de una crítica profunda la cual considera una serie de aspectos negativos que implica el turismo rural, principalmente en lo referente a lo económico, social y ambiental como se muestra en la tabla 4. Asimismo, se crea una alta dependencia del TR, descuidando la producción primaria como es la agricultura y ganadería fuente de supervivencia de la mayoría de las comunidades en México.

\section{Tabla 4}

Aspectos negativos del turismo rural

\begin{tabular}{|c|c|c|}
\hline Factores & Aspectos negativos & Causas \\
\hline ECONOMICO & $\begin{array}{l}\text { - Incremento de los precios de } \\
\text { los bienes y servicios. } \\
\text { - Incremento del valor de la } \\
\text { tierra }\end{array}$ & $\begin{array}{l}\text { - Incremento de la inversión y el } \\
\text { gasto } \\
\text { - } \quad \text { Mayor flujo de personas }\end{array}$ \\
\hline
\end{tabular}




\section{Tabla 4}

Continuación...

\begin{tabular}{|l|ll|l|}
\hline \multicolumn{1}{|c|}{ Factores } & \multicolumn{2}{|c|}{ Aspectos negativos } & \multicolumn{1}{c|}{ Causas } \\
\hline SOCIOCULTURAL & $\bullet$ & Cambia la cultura & $\bullet \begin{array}{l}\text { Diversidad de personas } \\
\text { visitantes. }\end{array}$ \\
\hline ECOLOGICO & $\bullet$ & Incrementa la delincuencia & \\
& $\bullet$ & $\begin{array}{l}\text { Incremento del ruido } \\
\text { contaminación }\end{array}$ & $\bullet \begin{array}{l}\text { Construcción de instalaciones } \\
\text { Eacilidades de acceso }\end{array}$ \\
\hline
\end{tabular}

Fuente: Elaboración propia con información de Shu-Tsu, 2010.

Adicional a lo anteriormente expuesto, en un estudio conceptual realizado por Tsephe y Eyono (2013) además de determinar los beneficios que deja el TR y que se han mencionado en diversos trabajos de este tema como es la creación de empleos principalmente, se pudieron identificar una serie de impactos negativos que afectan seriamente el desarrollo del TR como se puede apreciar en la tabla 5 .

\section{Tabla 5}

\section{Impactos negativos del turismo rural}

- Los habitantes de una comunidad tienden a ser explotados

- Los inversionistas externos se llevan la mayor parte de las utilidades

- Muchos alimentos y bebidas son llevadas de zonas urbanas para reducir costos.

- Aunque se generan empleos la mayora son mal pagados.

- Los productos y artesanías locales bajan de precio por la competencia entre los mismos habitantes.

- Se impacta el medio ambiente con la sobreexplotación de los recursos naturales.

- La falta de planeación adecuada del TR afecta a los habitantes de las comunidades. 
De igual forma en un estudio de análisis FODA realizado por Zhang (2012) en la provincia de Suzhou en China, se pudieron determinar una serie de desventajas que afectan directamente al desarrollo del
TR como se puede apreciar en la tabla 6. Estas desventajas muy bien pueden decirse que se pueden ver en muchas comunidades rurales de México.

\section{Tabla 6}

Desventajas del turismo rural

\begin{tabular}{|c|c|}
\hline Desventaja & Causas \\
\hline Inadecuada infraestructura & $\begin{array}{l}\text { - } \text { Falta de apoyo financiero } \\
\text { - } \quad \text { Falta de accesos adecuados. } \\
\text { - } \text { Transportación } \\
\text { - Alojamiento } \\
\text { - Salud }\end{array}$ \\
\hline Deficiente comercialización & $\begin{array}{l}\text { - } \\
\text { - } \quad \text { Lo carece de una imagen de destino } \\
\text { - La comunidad y sus habitantes carecen de } \\
\text { conocimientos en marketing. }\end{array}$ \\
\hline Falta de planeación y gestión turística & $\begin{array}{l}\text { - } \quad \text { Carencia de profesionales en el turismo rural } \\
\text { - } \quad \text { Falta de diseño de estrategias de marketing. } \\
\text { - Falta de formación a los habitantes de las } \\
\text { comunidades. }\end{array}$ \\
\hline Baja calidad de productos turísticos rurales & $\begin{array}{l}\text { - Falta de capacitación en la elaboración de productos } \\
\text { y servicios. } \\
\text { - } \quad \text { Falta de herramientas y maquinaria adecuada. } \\
\text { - } \quad \text { Falta de capacitación en el servicio. }\end{array}$ \\
\hline
\end{tabular}

Fuente: Elaboración propia con información de Zhang 2012. 
Aunado a lo anterior y a pesar que gobierno en los últimos años ha llevado a cabo diversos programas en aras del turismo alternativo y firmado un convenio con 14 organismos federales para fomentar el turismo rural para poder mejorar la forma de vivir de comunidades rurales, esto no ha sido posible debido a la falta de políticas concretas así como de una estructura administrativa y jurídica adecuada que permitan lograr el propósito principal del turismo rural que es mejorar la forma de vida de las personas en las comunidades marginadas en México (López y Palomino, 2012). Todo esto permite establecer que la actividad del turismo rural y los programas gubernamentales no necesariamente se puede considerar un factor de desarrollo en las comunidades rurales.

\section{Controversias del turismo}

El turismo en México es considerado una fuente importante de ingresos debido principalmente a toda una serie de elementos con que cuenta el país, lo que permitido el impulso del turismo considerando los aspectos social, económico y principalmente ambiental, y la mega diversidad de productos turísticos como es el turismo de naturaleza donde se contemplan: el ecoturismo, el turismo rural, turismo de aventura principalmente. Esto, más otros aspectos como es la calidez de su gente y la tranquilidad del campo, son aspectos que valoran por los promotores del turismo. Esto ha permitido a México estar entre los principales países que generan riqueza con el turismo (Ibáñez, 2011), ya que en 2019 los ingresos por turismo crecieron en un $10.2 \%$ y siendo visitado por 44.7 millones de turistas extranjeros (Reyna, 2020), y hasta el 2018 ocupaba el séptimo lugar a nivel mundial de acuerdo al parámetro de la Organización Mundial del Turismo (OMT, 2018). En este sentido se pueden mencionar algunos estados donde el turismo representa una importante fuente de ingresos como: Oaxaca, Chiapas, Campeche y Guerrero.

Sin embargo, de acuerdo a las cifras que maneja CONEVAL, los estados mencionados anteriormente son los que tienen mayor porcentaje de pobreza hasta el año 2018 como se muestra en la tabla 7.

\section{Tabla 7}

\section{Porcentaje de pobreza en México}

\begin{tabular}{|c|r|r|r|r|r|r|}
\hline \multirow{2}{*}{ Entidad federativa } & & \multicolumn{5}{|c|}{ Porcentaje } \\
\cline { 2 - 7 } & 2008 & 2010 & 2012 & 2014 & 2016 & 2018 \\
\hline Aguascalientes & 37.6 & 38.1 & 37.8 & 34.8 & 28.2 & 26.2 \\
\hline Baja California & 26.0 & 31.5 & 30.2 & 28.6 & 22.2 & 23.3 \\
\hline
\end{tabular}


Tabla 7

Continuación...

\begin{tabular}{|l|r|r|r|r|r|r|}
\hline \multirow{2}{*}{ Entidad federativa } & & \multicolumn{5}{|c|}{ Porcentaje } \\
\cline { 2 - 7 } & 2008 & 2010 & 2012 & 2014 & 2016 & 2018 \\
\hline Baja California Sur & 21.4 & 31.0 & 30.1 & 30.3 & 22.1 & 18.1 \\
\hline Campeche & 45.9 & 50.5 & 44.7 & 43.6 & 43.8 & 46.2 \\
\hline Coahuila & 32.7 & 27.8 & 27.9 & 30.2 & 24.8 & 22.5 \\
\hline Colima & 27.4 & 34.7 & 34.4 & 34.3 & 33.6 & 30.9 \\
\hline Chiapas & 77.0 & 78.5 & 74.7 & 76.2 & 77.1 & 76.4 \\
\hline Chihuahua & 32.1 & 38.8 & 35.3 & 34.4 & 30.6 & 26.3 \\
\hline Ciudad de México & 27.6 & 28.5 & 28.9 & 28.4 & 27.6 & 30.6 \\
\hline Durango & 48.4 & 51.6 & 50.1 & 43.5 & 36.0 & 37.3 \\
\hline Guanajuato & 44.1 & 48.5 & 44.5 & 46.6 & 42.4 & 43.4 \\
\hline Guerrero & 68.4 & 67.6 & 69.7 & 65.2 & 64.4 & 66.5 \\
\hline Hidalgo & 55.2 & 54.7 & 52.8 & 54.3 & 50.6 & 43.8 \\
\hline Jalisco & 36.7 & 37.0 & 39.8 & 35.4 & 31.8 & 28.4 \\
\hline México & 48.8 & 43.2 & 45.5 & 52.3 & 49.3 & 47.9 \\
\hline Michoacán & & 54.7 & 54.4 & 59.2 & 55.3 & 46.0 \\
\hline Morelos & & & & & & \\
\hline
\end{tabular}


Tabla 7

Continuación...

\begin{tabular}{|l|r|r|r|r|r|r|}
\hline \multirow{2}{*}{ Entidad federativa } & & \multicolumn{5}{|c|}{ Porcentaje } \\
\cline { 2 - 7 } & 2008 & 2010 & 2012 & 2014 & 2016 & 2018 \\
\hline Nayarit & 41.7 & 41.4 & 47.6 & 40.5 & 37.5 & 34.8 \\
\hline Nuevo León & 21.4 & 21.0 & 23.2 & 20.4 & 14.2 & 14.5 \\
\hline Oaxaca & 61.8 & 67.0 & 61.9 & 66.8 & 70.4 & 66.4 \\
\hline Puebla & 64.6 & 61.5 & 64.5 & 64.5 & 59.4 & 58.9 \\
\hline Querétaro & 35.2 & 41.4 & 36.9 & 34.2 & 31.1 & 27.6 \\
\hline Quintana Roo & 33.7 & 34.6 & 38.8 & 35.9 & 28.8 & 27.6 \\
\hline San Luis Potosí & 50.9 & 52.4 & 50.5 & 49.1 & 45.5 & 43.4 \\
\hline Sinaloa & 32.4 & 36.7 & 36.3 & 39.4 & 30.8 & 30.9 \\
\hline Sonora & 27.1 & 33.1 & 29.1 & 29.4 & 27.9 & 28.2 \\
\hline Tabasco & 53.8 & 57.1 & 49.7 & 49.6 & 50.9 & 53.6 \\
\hline Tamaulipas & 33.8 & 39.0 & 38.4 & 37.9 & 32.2 & 35.1 \\
\hline Tlaxcala & 59.6 & 60.3 & 57.9 & 58.9 & 53.9 & 48.4 \\
\hline Veracruz & 57.0 & 48.3 & 48.9 & 45.9 & 41.9 & 40.8 \\
\hline Yucatán & 60.2 & 54.2 & 52.3 & 49.0 & 46.8 \\
\hline Zacatecas & & & 58.0 & 62.2 & \\
\hline
\end{tabular}

Fuente: CONEVAL (2018). 
Aunado a lo anterior de acuerdo a SECTUR (2015), existe una rotación constante en las empresas turísticas de estos estados provocado principalmente por los bajos salarios que se perciben, lo cual influye en la baja calidad de vida de las personas.

\section{Metodología}

Para la realización de esta investigación conceptualreflexiva, se revisaron diversidad de artículos científicos y sitios web principalmente enfocados sobre la relación del turismo con la pobreza y el impacto que este tiene. De la igual forma se consultó información de la Secretaria de Turismo, organismo del gobierno de México cuyo objetivo es el desarrollo del turismo en todas sus acepciones.

\section{Resultados}

La pobreza representa un problema a nivel mundial, ya que no es exclusiva de los países de bajo desarrollo económico ya que también hace presencia en países altamente desarrollados, pero con las debidas proporciones. Esto ha provocado que las naciones instrumenten acciones encaminadas a reducir la pobreza mediante programas y políticas sociales que vayan en beneficio de las clases más vulnerables.

En México en cada periodo de gobierno se implementan programas de carácter social para enfrentar este problema sobre todo en el área rural. Pero en la mayoría de los casos estas acciones consisten en ayudas económicas y materiales que en nada contribuyen a reducir el índice de pobreza en México, en gran parte por el manejo incorrecto que se hace de los recursos de estos programas por parte de quienes son los responsables de ejecutarlos.

El turismo se ha considerado como una alternativa más para impulsar el desarrollo en las comunidades rurales mediante programas como son los pueblos mágicos, sin embargo, todavía quedan cientos de destinos rurales con recurso naturales que no son aprovechados para generar una fuente de ingresos mediante actividades turísticas. Debido principalmente a los intereses de grupos y personas que promueven el turismo, y que, si bien son generadores de empleos, estos son mal pagados y solo permiten que las personas contratadas que son habitantes de las comunidades solo puedan satisfacer sus necesidades básicas.

\section{Conclusiones}

A través de los años la situación precaria de las comunidades rurales en México ha traído como consecuencia toda una serie de necesidades de bienes y servicios, las cuales han sido satisfechas en menor medida por el gobierno, personas y organizaciones comerciales; sin embargo, esto no ha sido suficiente para reducir el rezago existente, a pesar del desarrollo de las diversas formas de comunicación que existen actualmente. En este sentido el Turismo juega un papel importante mediante la promoción de esta actividad aprovechando los recursos naturales, culturales y patrimoniales que caracterizan de forma distinta a cada comunidad.

Pero es claro que el Turismo a pesar de sus beneficios como es la generación de empleos e ingresos principalmente, no es la solución hasta ahora debido a los intereses personales y organizacionales cuando se desarrolla un proyecto turístico que por lo regular se realiza en regiones que tienen mayores atractivos y donde en la mayoría de los casos los habitantes no son incluidos en dichos proyectos. Por tal motivo el estado ha venido implementando toda una serie de regulaciones mediante programas sociales enfocadas al cuidado socioeconómico natural y cultural de las comunidades rurales incluyendo el concepto de sustentabilidad para la conservación principalmente de los recursos naturales, y 
además considerando el turismo en el desarrollo de proyectos que contribuyan la reducción del rezago que se tiene en muchas comunidades de México, mediante una oferta turística que permita en la medida que se pueda a lograr un crecimiento económico en las zonas rurales.

Si bien los diferentes programas sociales que el estado ha implementado a través de los años van encaminados a tratar de reducir el índice de la pobreza en México, estos no han dado los resultados esperados, pues a la fecha el estatus de millones de personas en estado de pobreza continua igual en virtud de que la ayuda que reciben a mediante las acciones de carácter social solo les permite subsistir ante el índice inflacionario que se refleja en el poder adquisitivo. En base a esta investigación, se puede considerar que el turismo rural en México no ha generado los resultados esperado para combatir la pobreza en el campo, en virtud de que la mayoría de los casos se enfatiza en la degradación ambiental y la comercialización de la cultura con beneficios económicos únicamente para los promotores del turismo sin considerar a los habitantes de las comunidades.

Sin embargo, a pesar de esto, el turismo sigue siendo una buena opción para reducir la pobreza en el ámbito rural en México, si se establecen los mecanismos apropiados que permitan implementar en forma adecuada programas que vayan encaminados al desarrollo rural sustentable, pero de forma incluyente. Esto es, que los habitantes de las comunidades participen directamente en el desarrollo de actividades turísticas y que puedan recibir beneficios económicos adicionales al sueldo que perciban. Existen muchas comunidades en México con un gran potencial de recurso naturales, culturales, patrimoniales, religiosos y tradicionales, que no han sido aprovechados como productos turísticos y que pudieran contribuir a mejorar la forma de vivir de las personas de una población rural mediante una apropiada comercialización, si se establecieran programas para tal propósito considerando lo mencionado en el párrafo anterior

\section{Referencias}

Adeyinka-Ojo, S. F., Khoo-Lattimore, C., y Nair, V. (2014). A Framework for Rural Tourism Destination Management and Marketing Organisations. Procedia Social and Behavioral Sciences, 144(1), 151-163.

Banco Mundial. (2015). Pobreza: Panorama General. http://www.bancomundial.org/es/topic/poverty/overvie $\mathrm{w \# 1}$

Benseny, G. (2007). El turismo en México apreciaciones sobre el turismo en espacio litoral. Aportes y Transferencias, 13-34.

Bruyere, B. L., Beh, A. W., y Lelengula, G. (2009). Differences in perceptions of communication, tourism benefits, and management issues in a protected area of rural Kenya. Environmental Management, 43(1), 4959.

Cardoso, C. (2006). Turismo Sostenible: una revisión conceptual aplicada. El Periplo Sustentable, Mayo, 521.

Cawley, M., y Gillmor, D. (2007). Integrated rural tourism: concepts and practice. Annals of Tourism Research, 35(2), 316-337. 
Colino, S. J. B., Gómez, C. M. A., y Martínez-Carrasco, P. F. (2014). Pobreza y políticas de desarrollo rural en México. Estudios Sociales (Hermosillo, Son.), 22(43), 9-35.

CONEVAL. (2018).

https://www.coneval.org.mx/Medicion/MP/Paginas/Po breza-2018.aspx

Connell, J., Page, y Bentley, T. (2009). Towards sustainable tourism planning in New Zealand: Monitoring local government planning under the Resource Management Act. Tourism Management, 30(6), 867-877.

Cortés, S. M. B., Juárez, S. J. P., y Ramírez, V. B. (2000). ¿Cómo se distribuyen los recursos para el combate a la pobreza? Análisis regional de la asignación del presupuesto del programa oportunidades en el estado de Puebla, México. Revista de Sociedad, Cultura y Desarrollo Sustentable, 7(1), 2011. Red Universidad Autónoma Indígena de México.

Diario Oficial de la Federación. (DOF). (2013). Secretaría de Agricultura, Ganadería.

Desarrollo Rural, Pesca y Alimentación. Cuarta Sección. Poder Ejecutivo. 13 de diciembre.

Díaz, F., y Fernández, C. (1995). Requerimientos Formativos en la Implantación de Actividades del Turismo Rural. Papers de Turisme, 19, 91-100.

Diéguez, M., Gueimonde, A., y Sinde-Cantorna, A. I. (2009). Turismo rural como estrategia de diversificación: factores determinantes y resultados en Galicia. (Spanish). Cuadernos De Gestión, 9(2), 31-53.
Fernández, M., García, M., y Picos, A. (2008). Un modelo estructural sobre la influencia de las motivaciones de ocio en la satisfacción de la visita turística. (Spanish). Revista De Psicología Del Trabajo y De Las Organizaciones, 24(2), 253-268.

Figueroa, E., Ramírez, O., González, J., Pérez, F., y Espinoza, L. (2012). Análisis del desempleo, la migración y la pobreza en México. Revista Mexicana de Agronegocios, 41(30), enero-junio, 2012, 835-847.

Fortanier, F., y Van, W. (2010). Sustainable tourism industry development in sub-Saharan Africa: Consequences of foreign hotels for local employment. International Business Review, 19(2), 191-205.

Forstner, K. (2004). Community ventures and access to markets: The role of intermediaries in marketing rural tourism products. Development Policy Review, 225, 497-514.

García, B. (2005). Características diferenciales del producto turismo rural. Cuadernos de Turismo, enerojunio, 113-133.

Garduño, M., Guzmán, C., y Zizumbo, L. (2009). Turismo rural: Participación de las comunidades y programas federales. El Periplo Sustentable, juliodiciembre, 5-30.

González, M. (2011). Una propuesta para desarrollar turismo rural en los municipios de Zacatecas, México: las rutas agro-culturales. PASOS Revista de Turismo y Patrimonio Cultural, Enero-Sin mes, 129-145.

Ghaderi, Z., y Henderson, J. (2012). Sustainable rural tourism in Iran: A perspective from Hawraman Village. Tourism Management Perspectives, 47-54. 
Guzmán, M., y Rebolloso, F. (2012). Turismo y sustentabilidad: paradigma de desarrollo entre lo tradicional y lo alternativo. (Spanish). Gestión $Y$ Estrategia, (41), 71-86.

Hunter, C. (2002). Sustainable tourism and the touristic ecological footprint. Environment, Development and Sustainability, 4, 7-20.

Ibáñez Pérez, R. (2011). Diagnóstico de la calidad y competitividad del sector turístico en México. Cuadernos de Turismo, Julio-Diciembre, 121-143.

Ibañes, R. (2015). Competitividad turística en México. TuryDes, 8(18).

Juárez, J., y Ramírez, B. (2007). El turismo rural como complemento al desarrollo territorial rural en zonas indígenas de México. Scripta Nova.

http://www.ub.edu/geocrit/sn/sn-236.htm

Lane, B. (2005). Sustainable Rural Tourism Strategies: A tool for development and conservation. RIAT Revista Interamericana de Ambiente y Turismo, 1(1).

Lee, T. H. (2013). Influence analysis of community resident support for sustainable tourism development. Tourism Management, 34, 37-46.

López-Guzmán, T., y Cañizares, S. (2009). Desarrollo socioeconómico de las zonas rurales con base en el turismo comunitario. Un estudio de caso en Nicaragua. (Spanish). Cuadernos De Desarrollo Rural, (62), 8197.

López, G., y Palomino, B. (2012). Guía de Apoyos Federales para el Desarrollo del Turismo de Naturaleza en México. Universidad Nacional Autónoma de México.

Lee, T. H. (2013). Influence analysis of community resident support for sustainable tourism development. Tourism Management, 34, 37-46.
Liu, Z. (2003). Sustainable tourism development: a critique. Journal of Sustainable Tourism, 11(6), 459475.

Liu, C.-H., Tzeng, G.-H., Lee, M.-H., y Lee, P.-Y. (2013). Improving metro-airport connection service for tourism development: Using hybrid MCDM models. Tourism Management Perspectives, 6, 95-107.

MacDonald, R., y Jooliffe, L. (2003). Cultural rural tourism evidence from Canada. Annals of Tourism Research, 30(2), 307-322.

Madrigal-Uribe, D., Cruz-Jiménez, G., RomeroContreras, T., Pérez-Ramírez, C., y ZizumboVillarreal, L. (2011). El turismo como intervención e implicaciones para las comunidades rurales. Gestión Turística, Julio-Diciembre, 229-264.

Mazaro, R., y Varzin, G. (2008). Modelos de Competitividad para Destinos Turísticos en el Marco de la Sostenibilidad. (Spanish). RAC - Revista De Administração Contemporânea, 12(3), 789-809.

Millán, M., López-Guzmán, T., y Agudo, E. (2006). El turismo rural como agente económico: desarrollo y distribución de la renta en la zona de Priego de Córdoba. CIRIEC-España, Revista de Economía Pública, Social y Cooperativa, agosto, 167-192.

Nolte, B. (2008). Sustainable tourism development in cross-border biosphere reserves of Central and Eastern Europe. In Cross-border Governance and Sustainable Spatial Development, 147-160. Springer Berlin Heidelberg.

Nunkoo, R., y Ramkissoon, H. (2011). Developing a community support model for tourism. Annals of Tourism Research, 38(3), 964-988.

Organización Mundial del Turismo. (2018). Ranking mundial del turismo.

http://www.datatur.sectur.gob.mx/SitePages/RankingO MT.aspx 
Ortiz, F., y Camargo, I. (2010). Propuesta de valores para una cultura organizacional en el turismo sostenible. PASOS Revista de Turismo y Patrimonio Cultural, 125-138.

Osorio, M. (2005). El entorno ambiental y el turismo sustentable, un análisis social. El Periplo Sustentable, Noviembre-Sin mes, 79-111.

Panyik, E., Costa, C., y Rátz, T. (2011). Implementing integrated rural tourism: An event-based approach. Tourism Management, 32(6), 1352-1363.

Pérez, A., Juárez, J., Ramírez, B., y Cesar, F. (2010). Turismo rural y empleo rural no agrícola en la Sierra Nororiente del estado de Puebla: caso red de Turismo Alternativo Totaltikpak, A.C. Investigaciones Geográficas (Mx), Abril, 57-71.

Pérez, S. (2010). El valor estratégico del turismo rural como alternativa sostenible de desarrollo territorial rural. Agronomía Colombiana, Septiembre-Diciembre, 507-513.

Petersen, L. (2010). An Examination of Integrated Rural Tourism Development in the Goris Region of Armenia. All Graduate Theses and Dissertations, 607.

Pinilla, H. (2009). Entre lo ideal y lo real; ¿los cambios en los enfoques propuestos de turismo rural sostenible desde la Organización de las Naciones Unidas contribuirían al desarrollo rural territorial? (Spanish). Cuadernos De Desarrollo Rural, (62), 145-171.

Prabhakaran, S., Nair, V., y Ramachandran, S. (2014). Community Participation in Rural Tourism: Towards a Conceptual Framework. Procedia - Social and Behavioral Sciences, 144, 290-295.
Ramírez, L. F. N., y de las Heras Pedrosa, C. (2013). Análisis y diseño de un sistema integral de gestión de calidad internacional como propuesta de aplicabilidad para las oficinas de convenciones en México. Encuentros Científicos-Tourism \& Management Studies, (1), 156-169. OCV.

Reyna, J. (2020). Ingresos por turismo. La Jornada. https://www.jornada.com.mx/2020/01/10/economia/02 1n1eco

Rigall-I-Torrent, R. (2008). Sustainable development in tourism municipalities: The role of public goods. Tourism Management, 29(5), 883-897.

Rodríguez, N., y Pallerols, G. (2003). El Turismo, un sector con futuro. (Spanish). Santiago, (100), 106-112. Rogel, I., Rojas, A. y Ortega, S. (2011). El turismo alternativo como estrategia de conservación de la reserva de la biosfera de la mariposa monarca (20082010). Quivera, Julio-Diciembre, 115-133.

Rodríguez Muñoz, G., y Acevedo Duarte, A. (2015). Cambios en la vida cotidiana de las mujeres a través de la incorporación al trabajo turístico en la Reserva de la Biosfera de la Mariposa Monarca. El Periplo Sustentable, 5-33.

Rogel, I., Rojas, A., y Ortega, S. (2011). El turismo alternativo como estrategia de conservación de la reserva de la biosfera de la mariposa monarca (20082010). Quivera, 13(2), julio-diciembre, 2011, 115-133.

Ruiz, D., y Barroso, M. (2011). Desarrollo rural, economía social y turismo rural: un análisis de casos. (Spanish). CIRIEC - España, Revista De Economía Pública, Social Y Cooperativa, (70), 55-80. 
Saarinen, J. (2006). Traditions of sustainability in tourism studies. Annals of Tourism Research, 33(4), 1121-1140.

Saxena, G., Clark, G., Oliver, T., e Ilbery, B. (2007). Conceptualizing integrated rural tourism. Tourism Geographies, 9(4), 347-370.

Saxena, G. (2008). Integrated rural tourism a border case study. Annals of Tourism Research, 35(1), 233-254.

Secretaría de Turismo (SECTUR). (2012). Sexto Informe de Labores. 2011-2012. Secretaría de Turismo del Gobierno Federal de México. www.sectur.gob.mx

SECTUR. (2015).

http://www.sectur.gob.mx/wpcontent/uploads/2015/02/PDF-Oaxaca.pdf

Serrano, R., Pérez, C., Manjarrez, É., y González, L. (2010). Turismo armónico como alternativa sustentable para una comunidad en el estado de México. Estudios y Perspectivas en Turismo, 970-993.

Sharpley, R. (2007). Flagship Attractions and Sustainable Rural Tourism Development: The Case of the Alnwick Garden, England, Journal of Sustainable Tourism, 15(2), 125-143.

Shu-Tzu, C. (2010). Rural tourism: perspectives from social exchange theory. Social Behavior \& Personality: An International Journal, 38(10), 1313-1322. doi:10.2224/sbp.2010.38.10.1313

Solsona, J. (2006). El turismo rural en Europa. Aportes y Transferencias, 25-35.

Solsona Monzonís, J. (2014). Análisis prospectivo del turismo rural: el caso de la Comunitat Valenciana. Cuadernos de Turismo, Julio-Diciembre, 313-334.
Strickland-Munro, J. K., Allison, H. E., y Moore, S. A. (2010). Using resilience concepts to investigate the impacts of protected area tourism on communities. Annals of Tourism Research, 37(2), 499-519.

Talaya, Á., Jiménez, J., Jiménez, J., y Santamaría, M. (2010). La gestión del turismo en los programas de innovación rural de Castilla-La Mancha. (Spanish). Cuadernos De Gestión, 10, 111-124. https://doi.org/10.5295/cdg.100192ae

Tao, T., y Wall, G. (2009). Tourism as a sustainable livelihood strategy. Tourism Management, 30(1), 9098.

Tarlombani, M. (2005). Turismo y sustentabilidad: Entre el discurso y la acción. Estudios y Perspectivas en Turismo, Septiembre-Sin mes, 222-238.

Tarlombani, M. (2005). Turismo y sustentabilidad: Entre el discurso y la acción. Estudios y Perspectivas en Turismo, 14(3), 222-238.

Touraya, K., y Junga, T. (2010). Exploratory Study on Contributions of ICTs to Sustainable Tourism Development in Manchester. Information and Communication Technologies in Tourism, 493-505.

Tsephe, N.P., y Eyono Obono, D. E. (2013). A Theoretical Framework for Rural Tourism Motivation Factors”, International Journal of Social, Management, Economics and Business Engineering, 7(1), 157-162.

Waligo, V. M., Clarke, J., y Hawkins, R. (2012). Implementing sustainable tourism: A multi-stakeholder involvement management framework. Tourism Management. Noviembre, 33(6), 1293-1616. 
Torres-Delgado, A., y López, P. F. (2012). The growth and spread of the concept of sustainable tourism: The contribution of institutional initiatives to tourism policy. Tourism Management Perspectives, 4, 1-10.

Vázquez, R. (2004). Turismo ecológico, turismo sustentable. (Spanish). Hospitalidad ESDAI, (6), 45-61.

Yasarata, M., Altinay, L., Burns, P., y Okumus, F. (2010). Politics and sustainable tourism development Can they co-exist? Voices from North Cyprus. Tourism Management, 31(3), 345-356.
Zhang, X., Song, H., y Huang, G. Q. (2009). Tourism supply chain management: A new research agenda. Tourism Management, 30(3), 345-358.

Zhang, X. (2012). Research on the Development Strategies of Rural Tourism in Suzhou Based on SWOT Analysis. Energy Procedia, 16, 1295-1299. 